METASTASIS

\section{It's nicer with DICER}

The expression level of microRNAs (miRNAs) is generally reduced in human cancers, but why this confers certain advantages to cancer cells is unknown. A group led by Stefano Piccolo has provided some answers to this question by identifying a new family of miRNAs that induces epithelial-mesenchymal transition (EMT) by inhibiting the expression of the microRNA regulator, DICER.

DICER processes miRNA precursors into mature miRNAs. These miRNAs control gene expression by binding to the $3^{\prime}$ untranslated region (UTR) of messenger RNAs (mRNAs). The authors identified an unusually long 3' UTR in mRNAs that encode DICER, which could mean that some miRNAs bind to this region and control DICER expression through a feedback mechanism. Analysis of miRNA binding sites in the $3^{\prime}$ UTR of DICER1 mRNA revealed binding of the miR-103/107 family. Accordingly, in multiple cell lines, expression of miR-103 or miR-107 reduced DICER expression and as a consequence mature miRNAs were generally downregulated. This inverse correlation between miR-103 and miR-107 and DICER was also observed in patients with breast cancer who were at an increased risk of developing metastases.

The authors sought to analyse the metastatic potential of the miR-103/107 family by overexpressing miR-107 in non-metastatic mouse cells and transplanting these cells into the cleared mammary fat pads of severe combined immunodeficient mice. After several weeks, these mice developed multiple micrometastases in the lung. Treatment of mice with antagomiR-103/107, an antisense oligonucleotide that silences miR-103 and miR-107, reduced metastatic colonization and restored levels of mature miRNAs. Similarly, overexpression of DICER in a highly metastatic cell line reduced the ability of xenografts to form metastases. An analysis of chromosomal arrays from breast cancers revealed that tumours with a reduced copy number of the DICER1 locus had an increased probability of developing metastases. Therefore, DICER might be a limiting factor in the metastatic process.
How does DICER inhibition by miR-103/107 promote metastasis? Piccolo and collaborators observed how the epithelial morphology of the immortalized mammary cell lines MCF10 and NMuMG switched to a more fibroblast-like morphology when miR-107 was overexpressed, indicating an EMT. The miR200 family are known to be required to suppress EMT. The levels of miR200 expression were increased on treatment with antagomiR-103/107, reverting the phenotypic and migratory effects that were triggered by miR-107.

These results suggest a new pathway by which DICER inhibition promotes a less differentiated phenotype that favours metastasis formation.

\section{Teresa Villanueva}

ORIGINAL RESEARCH PAPER Martello, G. et al. A MicroRNA targeting Dicer for metastasis control. Cell 141, 1195-1207 (2010)

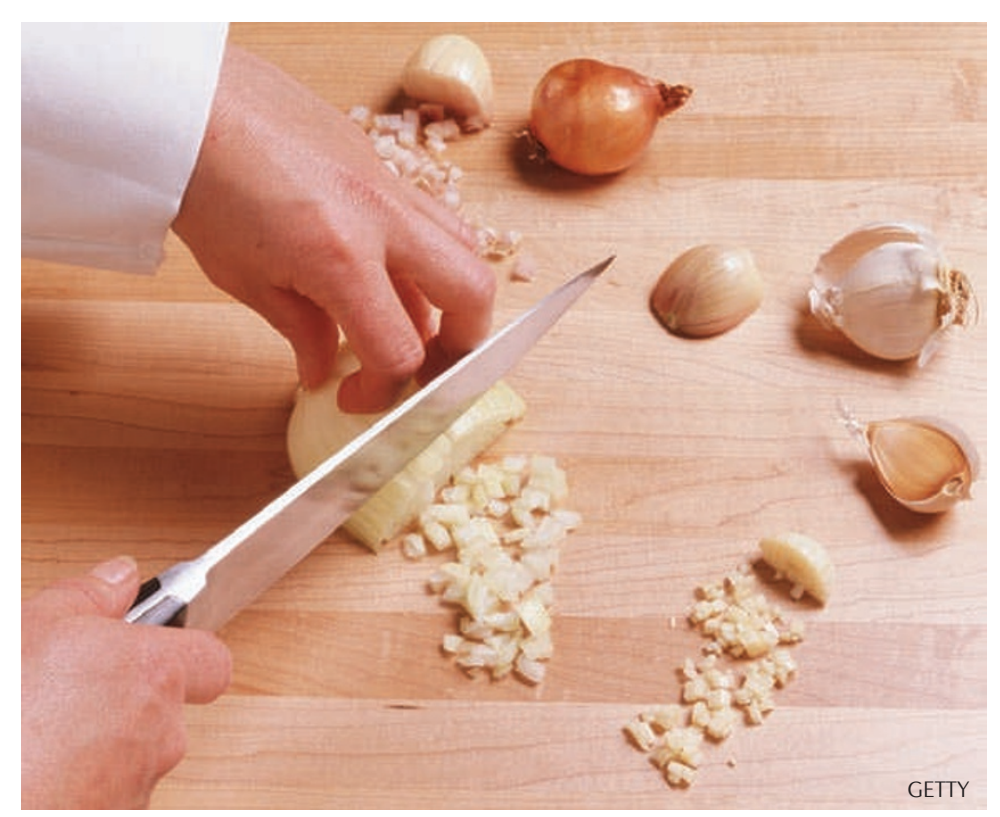

